\title{
Impulso al desarrollo de grupos vulnerables y marginados a través de apoyos económicos
}

Impulse to the development of vulnerable and margined groups through economic support

Luján Aguirre Ramón S. ${ }^{1 凶}$, Ortega Rodríguez Anabel ${ }^{1}$, Anchondo de las Casas Roberto O. ${ }^{2}$, Arizmendi Armendáriz Aldo E. ${ }^{2}$, Coronado Quintana José L. ${ }^{2}$

${ }^{1}$ Profesor de la Facultad de Ciencias Agrotecnológicas, Universidad Autónoma de Chihuahua Campus I, Chihuahua, Chih., México. Tel. (614)4391844

${ }^{2}$ Profesor de la Facultad de Contaduría y Administración, Universidad Autónoma de Chihuahua Campus II, Chihuahua, Chih., México. Tel (614)4420030.

$\bowtie$ Autor para correspondencia: rsaullujan@ hotmail.com

Recibido: 17/01/2016

Aceptado: 20/06/2016

\section{RESUMEN}

Derivado de las iniciativas gubernamentales, emergen programas que van más allá del asistencialismo, es decir, se busca detonar el emprendedurismo y autoempleo de grupos sociales que en el mayor de los casos se encuentran por debajo de la línea del bienestar que anualmente estima el Consejo Nacional de Evaluación de la Política de Desarrollo Social (CONEVAL, 2016). Como mecanismo de control y seguimiento a estos grupos sociales, los programas mismos contemplan una posterior y nueva inyección de recursos para detonar el empleo en las comunidades correspondientes a los citados grupos.

Palabras clave: vulnerabilidad, social, inclusión, apoyos, OSSE.

\begin{abstract}
Derived from governmental initiatives, programs emerge that go beyond welfare, ie seek to detonate entrepreneurship and self-employment of social groups that in the majority of cases are below the line of well-being that annually estimates the National Council Evaluation of the Social Development Policy (CONEVAL, 2016). As a mechanism to control and monitor these social groups, the programs themselves contemplate a subsequent and new injection of resources to detonate employment in the communities corresponding to the mentioned groups.
\end{abstract}

Keywords: vulnerability, social, inclusion, support, OSSE. 


\section{INTRODUCCIÓN}

Las particularidades de la economía actual, ponen a prueba las fortalezas que tienen los países para responder a las necesidades de su población, podemos citar como tales baluartes a la generación de fuentes de empleo, una objetiva distribución del ingreso y la generación de un Patrimonio Social.

Dentro de estas poblaciones se pueden identificar grupos vulnerables y/o marginados los cuales son considerados como uno de los Organismos del Sector Social de la Economía (OSSE) en el Catálogo de Organismos del Sector Social de la Economía 2016 que podrán acogerse y disfrutar de los apoyos y estímulos que otorga el Programa de Fomento a la Economía Social

En este sentido, la Secretaria de Desarrollo Social del Gobierno Federal basada en los programas de la cruzada contra el hambre, a través del Programa de Apoyo Alimentario (PAL) 2015, y el Programa Nacional para la Prevención Social de la Violencia y la Delincuencia (PRONAPRED) 2013, ha destinado una importante cantidad de recursos para que la población con alta marginación pueda acceder a apoyos para la promoción, fomento $\mathrm{y}$ fortalecimiento de los grupos vulnerables.

Tal es el caso de la Secretaría de Economía que por medio del Programa de Fomento a la Economía Social (2016) así como los programas regionales y especiales, ha atendido iniciativas productivas del Sector mediante el apoyo a proyectos productivos, la constitución, desarrollo, consolidación y expansión de Organismos del Sector y la participación en esquemas de financiamiento social.
El área de oportunidad es tan definida, que el Instituto de la Economía Social (INAES), órgano desconcentrado de la Secretaría de Economía, identifica como problema público que el Sector Social no se ha consolidado como una opción de inclusión productiva, laboral y financiera (INAES, 2016).

De acuerdo a la Ley de Economía Social y Solidaria (2013), se busca crear organismos de propiedad social, basado en relaciones de solidaridad, cooperación y reciprocidad, en los cuales se privilegian el trabajo y al ser humano, conformados y administrados en forma asociativa, para satisfacer las necesidades de sus integrantes y de sus comunidades, en resumen es la inclusión de aquellos segmentos de la población que se encuentran en desventaja y que enfrentan obstáculos para el acceso a oportunidades de inserción laboral, productiva y financiera.

El Programa implementa tres tipos de apoyo:

CRECE: Apoyos en efectivo y en especie o aportaciones que otorga el INAES para desarrollar capacidades organizativas y empresariales de los OSSE (Organismos del Sector Social de la Economía).

APOYOS PARA BANCA SOCIAL: Apoyos en efectivo o en especie, para fomentar la creación, transformación, fortalecimiento, consolidación e integración, de OSSE

INTEGRA: Apoyos en efectivo que se otorgan para proyectos productivos de los OSSE (Organismos del Sector Social de la Economía), ya sea para la ejecución de un proyecto productivo nuevo; para el desarrollo y consolidación de un proyecto en operación o para la vinculación en redes o cadenas de valor. 
Cuadro 1. Modalidades y montos de los apoyos del Programa.

\begin{tabular}{|c|c|c|}
\hline \multirow{2}{*}{ MODALIDADES } & \multicolumn{2}{|c|}{ MONTO MÁXIMO } \\
\cline { 2 - 3 } & OSSE COMO GRUPO SOCIAL & OSSE LEGALMENTE CONSTITUIDO \\
\hline 1.- EJECUCION DE UN PROYECTO PRODUCTIVO & HASTA 400 MIL PESOS & HASTA 1.0 MILLON DE PESOS \\
\hline NUEVO & HASTA 500 MIL PESOS \\
PROYECTO PRODUCTIVO EN OPERACIÓN & HASTA 1.5 MILLON DE PESOS \\
\hline $\begin{array}{c}\text { 3.- VINCULACION DE PROYECTOS PRODUCTIVOS } \\
\text { EN REDES O CADENAS DE VALOR }\end{array}$ & HASTA 600 MIL PESOS & HASTA 2.0 MILLONES DE PESOS \\
\hline
\end{tabular}

En este apartado el INAES apoya a especialmente a Grupos de Jóvenes (18 a 29 años), Mujeres o Grupos en situación de vulnerabilidad (discapacidad, adultos mayores, pobreza extrema, etc.), estos GRUPOS SOCIALES se pueden conformar con un mínimo de cinco integrantes, o se pueden integrar con la creación de grupos legalmente constituidos apegándose al catálogo de los Organismos del Sector social de la Economía. Los giros son variados, se maneja un catálogo que consta de más de 550 actividades, y van desde los agropecuarios, comercios, servicios, transporte, agroindustria, industria, minería, etc.
El apoyo se otorga para los siguientes conceptos.

Inversión Fija. - para adquisición de maquinaria, equipo de transporte, mobiliario y equipo, construcción de naves excepto para la adquisición de Terrenos o predios.

Inversión Diferida. - apoyo para pago del estudio de inversión, capacitación, asesoría técnica, acompañamiento y puesta en marcha de los proyectos.

Capital de Trabajo. - para adquisición de materia prima y suministros para la producción de bienes o servicios.

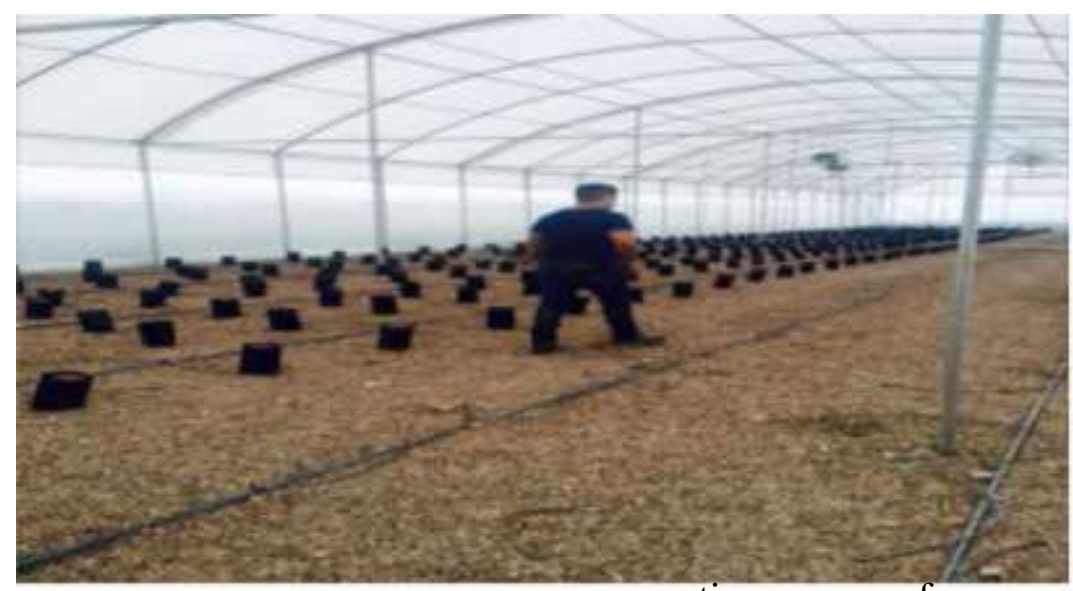

se tienen que sufragar por el interesado, lo cual Ilustración 1. Invernadero apoyado por INAES al OSSE INVERNORT en Chihuahua.

No obstante, la elaboración de un estudio de inversión, así como su gestión ante organismos de apoyo no está al alcance de toda la población, en el camino surgen necesidades que se traduce en un problema cuando no se cuentan con los medios necesarios. Es decir, se ha transformado en una oportunidad de negocio para terceros. 


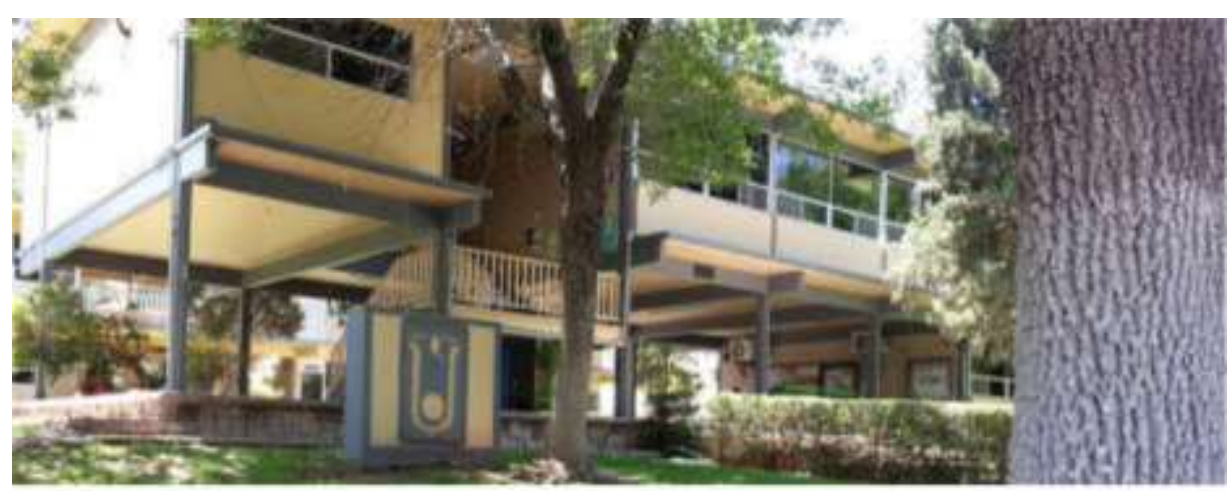

Ilustración. Facultad de Ciencias Agrotecnológicas de la Universidad Autónoma de Chihuahua.

Al respecto, la Facultad de Ciencias Agrotecnológicas de la Universidad Autónoma de Chihuahua (2014), durante los ejercicios 2014-2016, por medio de su Oficina de Administración de Proyectos, ha realizado estudios de inversión, capacitación, apoyo en la puesta en marcha, acompañamiento de los

Es importante señalar que existe al alcance de toda la población, sobre todo de los grupos de alta marginación, el acceso a recursos mediante apoyos, en donde las personas con una inquietud de iniciar con algún proyecto es necesario contactar a la Delegación del INAES de su entidad federativa. Así como posteriormente recibir asesoría y elaboración del estudio de inversión con consultores externos al servicio de los Organismos del Sector de la Economía, catedráticos de la Facultad de Ciencias Agrotecnológicas comisionados para ello. Se espera que este artículo sea de utilidad para aquellos

\section{LITERATURA CITADA}

Consejo Nacional de Evaluación de la Política de Desarrollo Social (CONEVAL), 2016. Líneas del bienestar. Consultado el 14 de diciembre de 2016. Disponible en: http://www.coneval.org.mx/Medicion/MP/Pagi nas/Lineas-de-bienestar-y-canasta-basica.aspx

Facultad de Ciencias Agrotecnológicas de la Universidad Autónoma de Chihuahua proyectos, así como asesoría técnica a las personas que integran tanto a los grupos sociales y como a los grupos legalmente constituidos, para que puedan acceder a los distintos apoyos económicos que se otorgan y poder iniciar la operación de proyectos productivos.

emprendedores que se quieran sumar a la generación del empleo y del desarrollo económico. Es importante mencionar que, en recursos otorgados como apoyo, el INAES da seguimiento, mediante visitas técnicas antes y después de autorizado el proyecto, es obligatorio comprobar física, normativa y fiscalmente la aplicación de los recursos, es decir, invertir, adquirir los conceptos solicitados, presentar facturas fiscales que avalen la adquisición de activos y en algunos casos comprobar la reintegración del recurso no ejercido.

(FACIATEC). 2014. Catálogo de Servicios. Consultado el 14 de diciembre 2016. Disponible en: http://www.faciatec.uach.mx/servicio/oficina_d e_administracion_de_proyectos/

Instituto Nacional de la Economía Social (INAES). 2016. Catálogo de Organismos del Sector Social de la Economía. Consultado el 14 
de diciembre de 2016. Disponible en: https://www.gob.mx/inaes/documentos/catalogo s-inaes

Instituto Nacional de la Economía Social (INAES). 2016. Reglas de Operación del Programa. Consultado el 14 de diciembre de 2016. Disponible en: http://www.gob.mx/inaes Ley de Economía Social y Solidaria (2013). Documentos. Consultado el 14 de diciembre de 2016. Disponible en:

https://www.gob.mx/inaes/documentos/ley-dela-economia-social-y-solidaria-36064 Programa de Apoyo Alimentario (PAL), 2015. Reglas de Operación del Programa. Consultado el 14 de diciembre de 2016. Disponible en: https://www.gob.mx/cms/uploads/attachment/fi le/70206/2015.pdf

Programa de Fomento a la Economía Social. 2016. Objetivos del Programa. Consultado el 14 de diciembre de 2016. Disponible en: http://www.gob.mx/sedesol/acciones-yprogramas/programa-de-fomento-a-laeconomia-social

Programa Nacional para la prevención social de la violencia y la delincuencia (PRONAPRED). 2013. Bases del Programa Nacional para la Prevención Social de la Violencia y la Delincuencia e Instalación de la Comisión Intersecretarial. Consultado el 14 de diciembre de 2016. Disponible en: http://www.gobernacion.gob.mx/archivosPortal /pdf/Bases120213.pdf 
Copyright (c) 2016 Ramón S. Luján Aguirre, Anabel Ortega Rodriguez, Roberto O. Anchondo de las Casas,

Aldo E Arizm endi Arm endáriz y José L Coronado Quintana

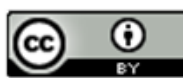

Este tex to está protegido por una licencia licencia Creative Commons 4 .0

Usted es libre para Compartir — copiar y redistribuir el material en cualquier medio o form ato- y Adaptar el documento — remezclar, transformar y crear a partir del material- para cualquier propósito,, incluso para fines comerciales, siempre que cumpla la condición de:

Atribución: Usted debe dar crédito a la obra original de manera adecuada, proporcionar un enlace a la licencia, e in dicar si se han realizado cam bios. Puede hacerlo en cualquier form a razonable, pero no de form a tal que sugiera que tiene el apoyo del licenciante o 10 recibe por el uso que hace de la obra.

Resumencielicencia - Textocompletodelalicencia 